\title{
Incidence of cerebral venous sinus thrombosis in adults in Germany - a retrospective study using health claims data
}

Josephine Jacob ( $\sim$ josephine.jacob@ingef.de)

InGef- Institute for Applied Health Research Berlin [Institut für angewandte Gesundheitsforschung Berlin GmbH) https://orcid.org/0000-0001-8769-9747

Robert Klamroth

Vivantes Klinikum im Friedrichshain - Klinik für Innere Medizin - Angiologie und Hämostaseologie

Tina Ploner

InGef- Institute for Applied Health Research Berlin [Institut für angewandte Gesundheitsforschung Berlin $\mathrm{GmbH}$ )

Thomas Harder

Robert Koch-Institute, Immunization unit

Judith Koch

Robert Koch-Institute, Immunization unit

Ole Wichmann

Robert Koch-Institute, Immunization unit

Jochen Walker

InGef- Institute for Applied Health Research Berlin [Institut für angewandte Gesundheitsforschung Berlin $\mathrm{GmbH}$ )

\section{Short Report}

Keywords: vaccination, cerebral venous sinus thrombosis, incidence, in-hospital mortality

Posted Date: April 19th, 2021

DOI: https://doi.org/10.21203/rs.3.rs-428469/v2

License: (c) (i) This work is licensed under a Creative Commons Attribution 4.0 International License.

Read Full License 
Incidence of cerebral venous sinus thrombosis in adults in Germany - a retrospective study using health claims data

Authors: Josephine Jacob ${ }^{1}$, Robert Klamroth², Tina Ploner ${ }^{1}$, Thomas Harder ${ }^{3}$, Judith Koch ${ }^{3}$, Ole Wichmann ${ }^{3}$, Jochen Walker ${ }^{1}$

${ }^{1}$ InGef- Institute for Applied Health Research Berlin [Institut für angewandte Gesundheitsforschung Berlin $\mathrm{GmbH}$ )

${ }^{2}$ Vivantes Klinikum im Friedrichshain - Klinik für Innere Medizin - Angiologie und Hämostaseologie

${ }^{3}$ Immunization Unit, Robert Koch Institute, Berlin

\section{Corresponding author:}

Josephine Jacob

InGef- Institut für angewandte Gesundheitsforschung Berlin GmbH Josephine.Jacob@ingef.de 


\begin{abstract}
In March 2021 several cases of cerebral venous sinus thrombosis (CVST) accompanied by thrombocytopenia were reported that occurred within two weeks after immunization with a viral vector-based COVID-19 vaccine. This study aimed to assess the background incidence of CVST and CVST with concurrent thrombocytopenia in adults using anonymized claims data from 2015-2019 in Germany. The average annual CVST incidence was 1.9 per 100,000 individuals $(95 \%-\mathrm{Cl} 1.4-2.3)$. It was higher in women aged 18 to 59 years as compared to same-aged men. In contrast to the currently reported immunization-related cases, CVST was rarely associated with concurrent thrombocytopenia or death.
\end{abstract}

\title{
Keywords
}

vaccination

cerebral venous sinus thrombosis

incidence

in-hospital mortality 


\section{Introduction}

Vaccinations play a key role in the prevention of infectious diseases and in the control of the current COVID-19 pandemic. Due to the limited number of participants in clinical trials it is difficult to identify adverse events that are very rare. With the rollout of a new vaccine, adverse events might occur which were previously unknown or incompletely reported in licensure studies. A timely detection and investigation of potentially related adverse events is critical for maintainance of confidence in the vaccination program. Claims data can support the identification and evaluation of vaccine safety signals in the post marketing phase by providing the background incidence of conditions potentially related to vaccine-related adverse events.

In March 2021 cases of cerebral venous sinus thrombosis (CVST), a rare form of a blood clot in the dural venous sinuses, were identified after immunization with a viral vector-based COVID-19 vaccine mainly in Europe. In Germany, 42 cases of CVST that occurred within 16 days after administration of the first dose of the AstraZeneca COVID-19 vaccine (ChAdOx1 nCov-19) were reported to the National Regulatory Authority as of 02 April 2021 (1). Among those, 23 were reported to be accompanied by thrombocytopenia and eight patients had died. At this point of time, approximately 2.9 million people had received ChAdOx1 nCov-19 in Germany.

Since CVST can occur independent from vaccination, the number of cases observed in a specific population with vaccination needs to be compared to the number of cases expected to occur without vaccination (i.e. the background incidence). However, epidemiological data regarding the background incidence of CVST is scarce worldwide and lacking for Germany (35). The aim of this study was to establish the background incidence of CVST and CVST with concurrent thrombocytopenia for various adult age-groups using anonymized claims data from Germany.

\section{Methods}

We used anonymized claims data from the InGef (InGef - Institute for Applied Health Research Berlin $\mathrm{GmbH}$ ) research database to conduct annual retrospective cohort studies to estimate 
CVST incidence per 100,000 persons for each year between 2015 to 2019 . The database includes anonymized longitudinal data from approximately eight million individuals insured in one of 60 German statutory health insurances (SHI) (mainly company or guild health insurances). The database has shown good accordance with the German population regarding measures of morbidity and mortality (6). For this study, a sample of four million insured individuals per year was drawn according to the age and sex distribution of the German population based on official national statistics (7). Approval by an institutional review board or Medical Ethics Committee was not required. To be included in the study patients had to be 18 years or older and continuously observable in the respective year or until death. CVST cases were defined as hospitalizations with a primary or secondary hospital discharge diagnosis of CVST (ICD-10-GM 167.6). CVST cases with coexisting thrombocytopenia were defined as cases with concurrently documented thrombocytopenia (ICD-10 GM D69.3, D69.4, D69.5 and D69.6) as a primary or secondary discharge diagnosis. The number of CVST patients who died during their CVST-related hospitalization was used to assess in-hospital mortality.

The incidence of CVST was calculated stratified by sex and age (age groups: 18-59 years; $\geq$ 60 years, $18-29$ years and $\geq 30$ years, $18-55$ years and $\geq 56$ years,) by dividing the number of CVST cases by the number of adult SHI insured persons in the InGef research database in the respective age and sex stratum in each study year. The mean incidence rate for the total period between 2015 and 2019 was calculated by estimating the mean number of cases per year and dividing it by the mean number of persons at risk per year. Incidence is presented per 100,000 persons with $95 \%$-confidence intervals $(95 \% \mathrm{Cl})$. The proportion of patients with concurrent thrombocytopenia and the in-hospital mortality were calculated relative to the total number of CVST patients per year.

\section{Results}

Approximately 3.2 million adult persons per year were included in the analysis. In this population, a mean annual CVST incidence of 1.9 per 100,000 individuals at risk $(95 \% \mathrm{Cl} 1.4-$ 2.3) (Table 1) was observed between 2015 to 2019. Extrapolated to the total German adult 
population this amounts to approximately 1,300 cases per year. CVST was more common in women aged 18 to 59 years as compared to same-aged men. In this age group, CVST incidence was 2.4 / 100,000 (95\%Cl 1.5-3.4) in women and $1.0 / 100,000(95 \% \mathrm{Cl} 0.4-1.7)$ in men. In individuals aged 60 years and older, CVST incidence was similar in women and men. Incidence estimates for various age-strata are shown in Table 1.

The number of individuals with CVST in combination with thrombocytopenia varied between 0 and 2 per year between 2015 and 2019. In the same period, the in-hospital mortality in CVST patients varied between $0 \%(n=0)$ in 2016 and 7.7\% $(n=4)$ in 2015 (Table S1). No differences between women and men or between different age groups were found regarding the concurrent occurrence of thrombocytopenia or in-hospital mortality (data not shown).

\section{Discussion}

Based on anonymized German claims data of about 3.2 million individuals, we estimated a CVST incidence of 1.9 per 100,000 persons at risk per year. Previous studies from Norway, the Netherlands and Australia reported CVST incidence rates of 1.3-1.8 / 100,000 persons at risk (3-5). In individuals aged 18 to 59 years, women were more than twice as likely to be treated due to CVST than men. Coutinho et al (4) also reported a higher CVST incidence in women between 31 and 50 years, whereas Devasagayam et al (5) did not find a higher risk in females of reproductive age.

In our study CVST cases with concurrent thrombocytopenia occurred very infrequently. Between 0 and 2 cases per year (i.e. up to $3 \%$ of the CVST cases) were observed in the study population. To our knowledge, no studies have previously reported population-based epidemiologic data on the background incidence of CVST with concurrent thrombocytopenia. The in-hospital mortality in this study varied between $0.0 \%$ and $7.7 \%$, which is in line with previously published mortality rates. Haghighi et al (8) reported a mortality of $4.4 \%$ and Coutinho and colleagues (9) showed that CVST mortality has declined over the years to less than $10 \%$ in 2010. As of 02 April 2021, 19\% $(n=8)$ of the patients with CSVT following immunization with ChAdOx1 nCov-19 had died in Germany (1). 
A limitation of our study is that the number of observed CVST with concurrent thrombocytopenia as well as the number of deceased patients were rather low. Hence the reported results must be interpreted with caution and could also reflect underreporting in clinical practice.

Our data are intended to support decision-makers and regulators in the vaccination field to identify and interpret safety signals and to decide on the best use of the recently licensed COVID19 vaccines. The observed accumulation of CVST cases within 16 days post ChAdOx1 nCov-19 vaccination in combination with the atypical presence of thrombocytopenia and high mortality strengthens the assumption that a part of the currently immunization-related CVST cases represents a previously unknown disease entity, which has been suggested to be called vaccine-induced immune thrombotic thrombocytopenia (VITT) (10). 


\section{Keypoints}

- The incidence of CVST was 1.9 per 100,000 persons at risk per year between 2015 and 2019.

- CVST was more common in women aged 18-59 years, than in same-aged men.

- The concurrent occurrence of CVST and thrombocytopenia was rare and CVSTrelated in-hospital mortality was low.

- The atypical presence of thrombocytopenia and the high mortality in immunizationrelated CVST cases strengthen the assumption that a part of these CVST cases represents a new disease entity (vaccine-induced immune thrombotic thrombocytopenia - VITT). 


\section{Funding / Acknowledgment}

The research leading to these results were generated within the project egePan-Unimed which received funding from the Federal Ministry of Education and Research under Grant Agreement No 01KX2021 as part of the Netzwerk Universitätsmedizin.

\section{Conflict of interest}

The authors have no conflicts of interest to declare that are relevant to the content of this article. 


\section{References}

1. Paul-Ehrlich-Institut. Sicherheitsbericht - Verdachtsfälle von Nebenwirlungen und Impfkomplikationen nach Impfung zum Schutz vor COVID-19 [Internet]. Langen; 2021 Apr [cited 2021 Apr 12] p. 20. Available from: https://www.pei.de/SharedDocs/Downloads/DE/newsroom/dossiers/sicherheitsberichte/ sicherheitsbericht-27-12-bis-02-04-21.pdf?_blob=publicationFile\&v=4

2. Koch-Institut R. Epidemiologisches Bulletin 16/2021. 2021;76.

3. Kristoffersen Espen Saxhaug, Harper Charlotte Elena, Vetvik Kjersti Grøtta, Zarnovicky Svetozar, Hansen Jakob Møller, Faiz Kashif Waqar. Incidence and Mortality of Cerebral Venous Thrombosis in a Norwegian Population. Stroke. 2020 Oct 1;51(10):3023-9.

4. Coutinho JM, Zuurbier SM, Aramideh M, Stam J. The incidence of cerebral venous thrombosis: a cross-sectional study. Stroke. 2012 Dec;43(12):3375-7.

5. Devasagayam Sharon, Wyatt Ben, Leyden James, Kleinig Timothy. Cerebral Venous Sinus Thrombosis Incidence Is Higher Than Previously Thought. Stroke. 2016 Sep $1 ; 47(9): 2180-2$.

6. Andersohn F, Walker J. Characteristics and external validity of the German Health Risk Institute (HRI) Database. Pharmacoepidemiol Drug Saf. 2016 Jan 1;25(1):106-9.

7. Statistisches Bundesamt Deutschland - 12411-0013: Bevölkerung: Bundesländer, Stichtag, Geschlecht, Altersjahre [Internet]. GENESIS-ONLINE. 2021 [cited 2021 Apr 9]. Available from: https://wwwgenesis.destatis.de/genesis//online?operation=table\&code=124110013\&bypass=true\&levelindex $=0 \&$ levelid $=1617953251928 \#$ abreadcrumb

8. Haghighi Afshin Borhani, Edgell Randall C., Cruz-Flores Salvador, Feen Eli, Piriyawat Paisith, Vora Nirav, et al. Mortality of Cerebral Venous-Sinus Thrombosis in a Large National Sample. Stroke. 2012 Jan 1;43(1):262-4. 
9. Coutinho Jonathan M., Zuurbier Susanna M., Stam Jan. Declining Mortality in Cerebral Venous Thrombosis. Stroke. 2014 May 1;45(5):1338-41.

10. Greinacher A, Thiele T, Warkentin TE, Weisser K, Kyrle PA, Eichinger S. Thrombotic Thrombocytopenia after ChAdOx1 nCov-19 Vaccination. New England Journal of Medicine [Internet]. 2021 Apr 9 [cited 2021 Apr 12]; Available from: http://www.nejm.org/doi/10.1056/NEJMoa2104840 
Table 1 CVST incidence per 100.000 persons at risk - stratified by age group and sex

\begin{tabular}{|c|c|c|c|c|}
\hline Age group & Sex & $\mathrm{N}$ at risk & $\mathbf{n}$ & $\begin{array}{l}\text { n / 100.000 } \\
(95 \% \mathrm{Cl})\end{array}$ \\
\hline \multirow[t]{3}{*}{ Adults ( $\geq 18$ years) } & female & $1,678,377$ & 38 & $2.3(1.5-3.0)$ \\
\hline & male & $1,584,009$ & 23 & $1.4(0.8-2.0)$ \\
\hline & total & $3,262,386$ & 61 & $1.9(1.4-2.3)$ \\
\hline \multirow[t]{3}{*}{18 - 59 years } & female & $1,024,324$ & 25 & $2.4(1.5-3.4)$ \\
\hline & male & $1,052,556$ & 11 & $1.0(0.4-1.7)$ \\
\hline & total & $2,076,880$ & 36 & $1.7(1.2-2.3)$ \\
\hline \multirow[t]{3}{*}{$\geq 60$ years } & female & 654,053 & 13 & $2.0(0.9-3.0)$ \\
\hline & male & 531,453 & 12 & $2.2(1.0-3.5)$ \\
\hline & total & $1,185,506$ & 25 & $2.1(1.3-2.9)$ \\
\hline \multirow[t]{3}{*}{18 to 29 years } & female & 230,954 & 6 & $2.6(0.5-4.7)$ \\
\hline & male & 245,967 & 2 & $0.8(0.0-1.9)$ \\
\hline & total & 476,921 & 8 & $1.7(0.5-2.8)$ \\
\hline \multirow[t]{3}{*}{$\geq 30$ years } & female & $1,447,424$ & 32 & $2.2(1.4-3.0)$ \\
\hline & male & $1,338,042$ & 21 & $1.6(0.9-2.2)$ \\
\hline & total & $2,785,465$ & 53 & $1.9(1.4-2.4)$ \\
\hline \multirow[t]{3}{*}{18 to 55 years } & female & 903,224 & 23 & $2.5(1.5-3.6)$ \\
\hline & male & 931,623 & 10 & $1.1(0.4-1.7)$ \\
\hline & total & $1,834,846$ & 33 & $1.8(1.2-2.4)$ \\
\hline \multirow[t]{3}{*}{$\geq 56$ years } & female & 775,154 & 15 & $1.9(1.0-2.9)$ \\
\hline & male & 652,386 & 13 & $2.1(1.0-3.3)$ \\
\hline & total & $1,427,540$ & 28 & $2.0(1.2-2.7)$ \\
\hline
\end{tabular}

$\mathrm{N}$ number of individuals at risk; $\mathrm{n}$ number of individuals with a hospital main or secondary discharge diagnosis of CVST; $\mathrm{n}$ / 100.000 incidence rate per 100.000 persons at risk; $95 \% \mathrm{Cl}$ - Confidenceintervall 
Table S1 - Number of CVST patients and a concurrent thrombocytopenia and number of patients deceased during a hospitalization with CVST per calendar year relative to the number of CVST events per calendar year

\begin{tabular}{lll}
\hline & $\begin{array}{l}\text { I67.6 (D69.3, D69.4, D69.5, D69.6) } \\
\text { n (\%) }\end{array}$ & $\begin{array}{l}\text { In hospital mortality } \\
\text { n (\%) }\end{array}$ \\
\hline 2015 & $1(1.9 \%)$ & $4(7.7 \%)$ \\
\hline 2016 & $0(0.0 \%)$ & $0(0.0 \%)$ \\
\hline 2017 & $0(0.0 \%)$ & $3(4.9 \%)$ \\
\hline 2018 & $2(3.1 \%)$ & $2(3.1 \%)$ \\
\hline 2019 & $1(1.4 \%)$ & $4(5.8 \%)$ \\
\hline
\end{tabular}

$\mathrm{n}$ number of patients; \% - proportion relative to the total number of CVST cases per year 